\title{
Painless Hematochezia Due to Appendicitis: Case Report and Review of Literature
}

\author{
Bin $\mathrm{Xu}^{1}$, Wen Pan ${ }^{2}$, Lifang $\mathrm{Zhao}^{2}$ and Sumei Sha ${ }^{3 *}$ \\ ${ }^{1}$ Chenggong Hospital of Xiamen University (Central Hospital of the 73rd Chinese People's Liberation Army), No. 94-96, Wen- \\ yuan Road, Xiamen, Fujian, China
}

${ }^{2}$ State Key Laboratory of Cancer Biology \& Xijing Hospital of Digestive Diseases, the Air Force Medical University, 127 Changle Western Road, Xi'an, Shaanxi Province, China

${ }^{3}$ Department of Gastroenterology, the Second Affiliated Hospital of Xi'an Jiaotong University, Shaanxi Provincial, Key Laboratory of Gastrointestinal Motility Disorders, Shaanxi Provincial, Clinical Research Center of Gastrointestinal Diseases, China

*Corresponding author: Sumei Sha, Department of Gastroenterology, the Second Affiliated Hospital of Xi'an Jiaotong University, Shaanxi Provincial, Key Laboratory of Gastrointestinal Motility Disorders, Shaanxi Provincial, Clinical Research Center of Gastrointestinal Diseases, China

ARTICLE INFO

Received: 慧 October 02, 2020

Published: 幽 October 13, 2020

Citation: Bin Xu, Wen Pan, Lifang Zhao, Sumei Sha. Painless Hematochezia Due to Appendicitis: Case Report and Review of Literature. Biomed J Sci \& Tech Res 31(1)2020. BJSTR. MS.ID.005054.

Keywords: Lower Gastrointestinal Bleeding; Appendiceal Hemorrhage; Appendicitis; Case Report

\begin{abstract}
Background: Lower gastrointestinal bleeding arising from the appendix is an extremely rare condition.

Case Presentation: We report a case of appendiceal hemorrhage in a middleaged male. Colonoscopy showed active bleeding from the orifice of the appendix. After endoscopic hemostasis failed, the patient underwent appendectomy. Subsequent histologic evaluation revealed evidence of acute inflammatory infiltrate. He recovered well after appendectomy.
\end{abstract}

Conclusions: Bleeding of appendiceal origin is very rare, but it should be considered during differential diagnosis of lower gastrointestinal bleeding. Appendectomy would be the most effective therapy for bleeding from the appendix.

Abbreviations: CT: Computed Tomography; IBD: Inflammation Bowel Disease

\section{Background}

Lower gastrointestinal bleeding, hemorrhage from the source distal to the ligament of Treitz, is a common emergency in gastroenterology, with an estimated incidence of 33/1000001. Lower gastrointestinal bleeding is causes for lower gastrointestinal bleeding include diverticular disease, vascular ectasia, ischemic, inflammatory or infectious colitis, colonic neoplasia (including post polypectomy bleeding), anorectal causes (including haemorrhoids, fissures and rectal varices), and small bowel lesions (Crohn's, vascular ectasia, Meckel's diverticula, and small bowel tumors). Despite improvements in diagnostic tools and techniques, survey of a bleeding source is a challenging problem in modern medical practice for patients with lower gastrointestinal bleeding. The bleeding source could not be identified even with extensive evaluation in $10 \%$ of patients2. Lower gastrointestinal bleeding arising from the appendix is an extremely rare condition. Herein we report a rare case of painless hematochezia due to appendicitis in a middle-aged male.

\section{Case Presentation}

A 46-year-old man was admitted to our department on an emergency basis because of a sudden onset of hematochezia. The patient had neither fever nor abdominal pain. He denied prior episodes of diarrhea and melena. He denied being nauseous or vomitous and experiencing hematemesis. He had a past history of melena and hematochezia 1 year earlier. At that time, computed tomography (CT) scan only found a slight thickening of the terminal ileum, but no lesion was found in gastroscopy, colonoscopy or enteroscopy. He was discharged after medical treatment at that time [1]. He had no significant medication history and family history. 
At admission, the patient's vital signs were within normal ranges. No abnormal findings were revealed throughout physical examination and laboratory tests except slightly decreased hemoglobin (from 17.4 to $13.3 \mathrm{~g} / \mathrm{dL}$ within 2 days). On the abdominopelvic computer tomography scan, we noted mild wall thickening in the terminal ileum/appendix. Colonoscopy showed dark blood fill without definitive evidence of bleeding foci in the entire colon or rectum and revealed that active bleeding originated from the orifice of the appendix Figure 1. No blood clots were found in the proximal part of the ileocecal valve. [2] The distal ileum, which was also viewed directly, appeared normal. Endoscopic hemostasis was failure, local injection of epinephrine did not stop bleeding. Urgent surgical management for the arrest of continuous hemorrhage was performed. The small intestine and colon appeared normal during surgical exploration, and a slight swelling of the appendix, hence appendectomy was performed uneventfully. Macroscopically, the resected appendix measured $12 \mathrm{~cm}$ in length. The appendix appeared grossly to be mildly edematous and hyperemic on the serosal surface and with much blood-filled in.
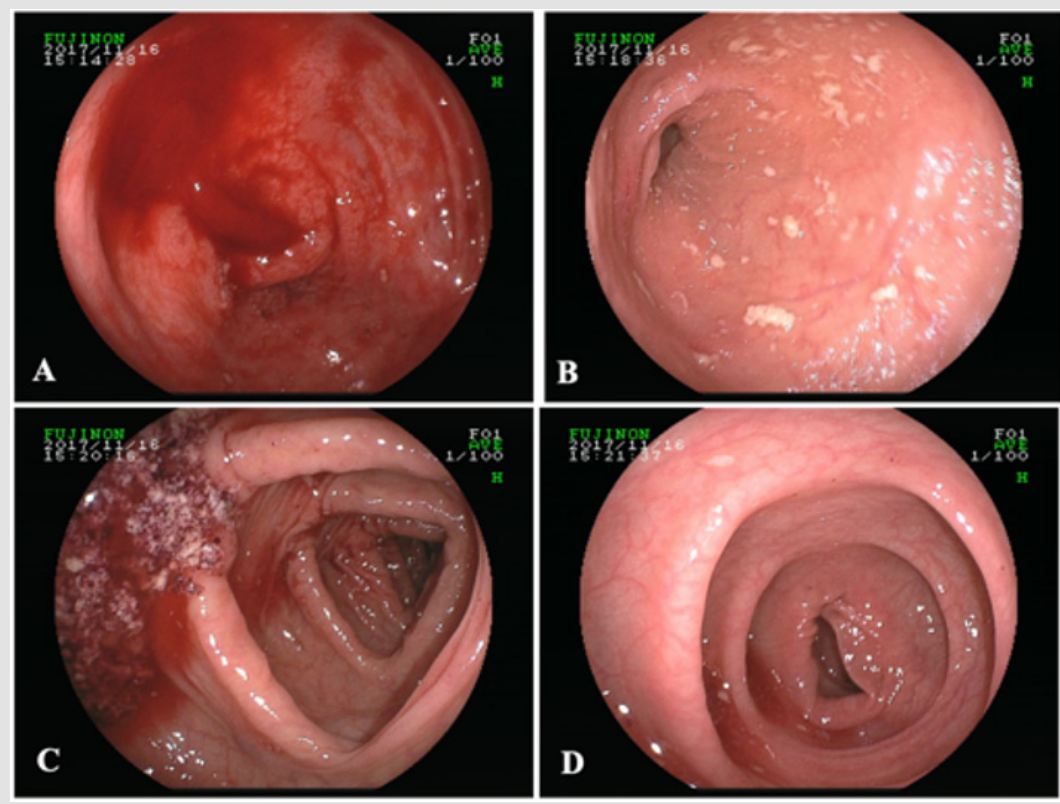

Figure 1: The colonoscopic finding showed active bleeding from the appendiceal orifice. A: Opening of appendix, B: terminal ileum, C: ascending colon, D: sigmoid colon.

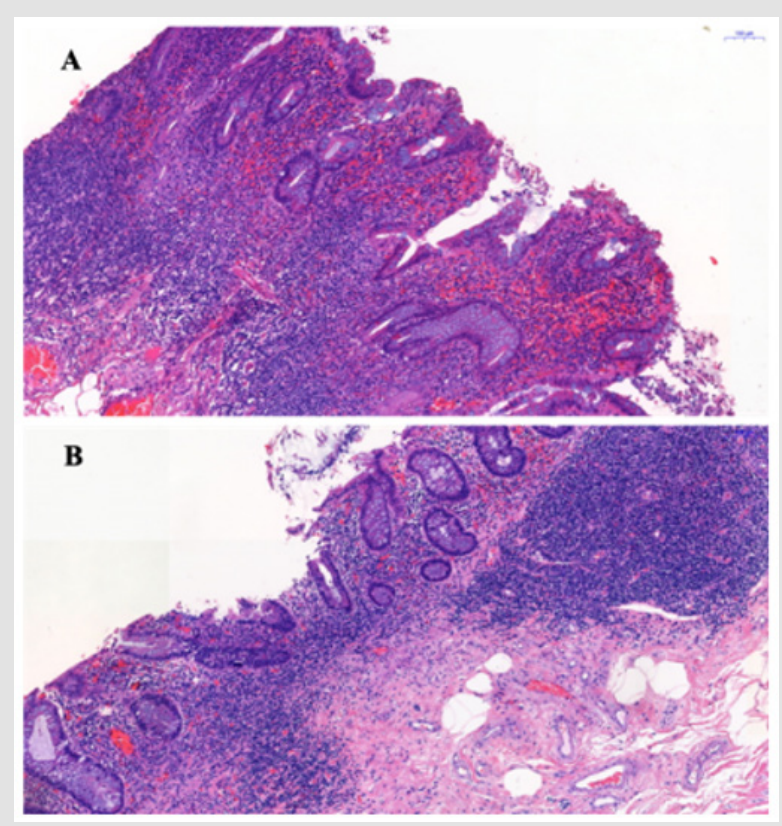

Figure 2: H\&E staining showed acute attack of chronic appendicitis with hyperemia and hemorrhage. The microscopic findings showed erosion of the mucosa with hemorrhage. Large number of inflammatory cells infiltrate in the submucosa. 
On cut, mucosa rough, erosion with bleeding was noted in the lumen of the root and apex appendix. Microscopically, the histology examination of the appendix revealed erosion of the mucosa with hemorrhage. There was no granuloma formation or vascular lesion over the entire appendix Figure 2. The post-operative course went smoothly, and the patient was discharged 5 days later. There was no recurrence of hematochezia after 24 months' postoperative followup.

\section{Discussion and Conclusions}

Lower gastrointestinal bleeding of appendiceal origin is extremely rare, and only a few case reports have been published in the English literature. Several pathologic conditions have been reported to be related to appendiceal bleeding, including inflammation bowel disease (IBD) [3,4] appendicitis [5], intussusceptions [6], gastrointestinal stromal tumor [7], diverticulitis [8,9] angiodysplasia [10], neoplasm, endometriosis [11] and erosion of appendiceal mucosa [12,13]. Besides, chemotherapy-induced ulceration, ischemic ulceration, parasitic ulceration, and angiomyolipoma also should be considered in differentiation diagnoses. Bleeding from the appendix with any cause, especially identified as the direct visualization at the time of colonoscopy is quite rare. Appendicitis leading to appendiceal hemorrhage is also unusual. In our case, the size and nature of appendix looked normal, but the histologic examination revealed hemorrhagic findings with several erosive lesions and surrounding inflammatory infiltrates mostly at mucosal layer without transmural inflammations. In other words, appendiceal bleeding should be suspicious when acute of chronic recurrent lower gastrointestinal bleeding.

Various diagnostic tools, such as barium-enema, colonoscopy, mesenteric artery angiography, and abdominal CT scan, are used for the diagnosis of appendiceal bleeding. Among them, colonoscopy is the single most frequent intervention in evaluating all the patients with lower GI bleeding, with low morbidity and mortality rates. In 2009, [14] reviewed 20 reported cases of appendiceal hemorrhage. They found bleeding from the appendix was identified by preoperative CT and/or colonoscopy in 11 cases, as in our case. 5 cases were diagnosis by barium enema. In only 2 cases definitive diagnosis was made using mesenteric angiography. [13] cases of hemorrhage from the appendix, the bleeding from the appendiceal orifice could be identified in 5 cases5. Since the multidetector CT was proven to be quite valuable in evaluating gastrointestinal bleeding, [12] speculated the combination of colonoscopy with abdominal CT may increase diagnostic accuracy. In our case, CT scan only found a slight thickening of the terminal ileum, we also saw bleeding through the colonoscopy.

The treatment of appendiceal hemorrhage is individualized and depends on the underlying pathologic anomaly and the patient's general condition. Colonoscopy hemostasis can be used in certain cases of lower gastrointestinal bleeding, there has been two successful case report regarding colonoscopy treatment of appendiceal bleeding until now. In 2010, Il [15] reported two cases of the use of colonoscopic clipping to treat appendiceal bleeding for the first time. In 2011, Il [16] successfully performed endoscopic clipping without any complications in a patient with acute appendiceal bleeding after confirming that there was no evidence of acute appendicitis or other specific lesions around appendix on abdominal CT and colonoscopy. Surgical treatment plays an important role in the management of appendiceal bleeding. The conventional or laparoscopic appendectomy is known to be the most common treatment for appendiceal bleeding based on a review of the literature to date. Appendectomy had been also performed in 9 of the 13 previous cases with appendiceal bleeding in a review [5].

More extensive resection such as cecectomy, ileocecectomy or right hemicolectomy has been reported to manage appendiceal bleeding according to findings during the operation. [17] reported their experience of temporary hemostasis in a patient with angiodysplasia of the appendix with percutaneous transcatheter arterial embolization using permanent material (micro coil) in 2001. Unfortunately, massive bleeding recurred two weeks after embolization. The patient subsequently underwent conventional appendectomy. Our case was successfully treated with conventional appendectomy. Although lower gastrointestinal bleeding of appendiceal origin is very rare, benign appendiceal lesions, such as ulcer or erosion, should be considered during differential diagnosis of lower gastrointestinal bleeding. It was difficult to control the bleeding from the appendiceal orifice with endoscopic therapy, appendectomy would be the most effective therapy for bleeding from the appendix.

\section{Declarations}

\section{Funding}

This study was supported by grants from the National Natural Science Foundation of China (Nos. 81500423 and 81502082). The funding bodies had no role in the study design, collection, analysis or interpretation of the data.

\section{References}

1. Lanas A, Garcia-Rodriguez LA, Polo-Tomas M, Ponce M, Alonso-Abreu I, et al. (2009) Time trends and impact of upper and lower gastrointestinal bleeding and perforation in clinical practice. Am J Gastroenterol 104(7): 1633-1641.

2. Imdahl A (2001) Genesis and pathophysiology of lower gastrointestinal bleeding. Langenbecks Arch Surg 386(1): 1-7.

3. Geerken RG, Gibbons RB. (1974) Isolated Crohn's disease of the appendix: case report. Mil Med 139(3): 215-217.

4. Brown WK, Peters RW (1976) Crohn's disease of the appendix presenting as lower intestinal hemorrhage and cecal mass. Am J Gastroenterol 65(4): 349-352. 
5. Yamazaki K, Nakao K, Tsunoda A, Nozomi Yoshikawa, Michio Imawari, et al. (2006) Successful laparoscopic treatment of hemorrhage from the appendix with phlegmonous acute appendicitis: a case report and review of the literature. Gastrointest Endosc 63(6): 877-880.

6. Jevon GP, Daya D, Qizilbash AH (1992) Intussusception of the appendix. A report of four cases and review of the literature. Arch Pathol Lab Med 116: $960-964$.

7. Kim KJ, Moon W, Park MI, Park SJ, Lee SH, et al. (2007) Gastrointestinal stromal tumor of appendix incidentally diagnosed by appendiceal hemorrhage. World J Gastroenterol 13(23): 3265-3267.

8. Norman DA, Morrison EB, Meyers WM Jr (1980) Massive gastrointestinal hemorrhage from a diverticulum of the appendix. Dig Dis Sci 25: 145147.

9. Ogawa Y, Asayama N, Nagata S (2018) Acute gastrointestinal bleeding from appendiceal diverticulitis diagnosed preoperatively by combined short-interval computed tomography and colonoscopy: A case report Dig Endosc 30(3): 392-394.

10. Choi JM, Lee SH, Lee SH, Ahn Bk, Baek SU, et al. (2016) Hematochezia due to Angiodysplasia of the Appendix. Ann Coloproctol 32(3): 117-119.

\section{ISSN: 2574-1241}

DOI: 10.26717/BJSTR.2020.31.005054

Sumei Sha. Biomed J Sci \& Tech Res

(C) (P) This work is licensed under Creative

Submission Link: https://biomedres.us/submit-manuscript.php
11. Emre A, Akbulut S, Yilmaz M (2013) An unusual cause of acute appendicitis: Appendiceal endometriosis. Int J Surg Case Rep 4: 54-57.

12. Chiang CC, Tu CW, Liao CS (2011) Appendiceal hemorrhage -- an uncommon cause of lower gastrointestinal bleeding. J Chin Med Assoc 74(6): 277-279.

13. Chung KS, Gao JP (2010) Massive lower gastrointestinal bleeding from the appendix. Gut Liver 5: 234-237.

14. Baek SK, Kim YH, Kim SP (2010) Acute lower gastrointestinal bleeding due to appendiceal mucosal erosion. Surg Laparosc Endosc Percutan Tech 20(3): e110-113.

15. Park I, Kwon CI, Ko KH, Sung Pyo Hong, Pil Won Park et al. (2010) Colonoscopic clipping as a treatment for appendiceal bleeding. Gut Liver 4(3): 411-414.

16. Chung IH, Kim KH (2011) A case of successful colonoscopic treatment of acute appendiceal bleeding by endoclips. J Korean Soc Coloproctol 27(6): 329-332.

17. Kyokane T, Akita Y, Katayama M, Kitagawa Yoshimi, Sato Taichiro, et al. (2001) Angiodysplasia of the appendix. Am J Gastroenterol 96(1): 242244.

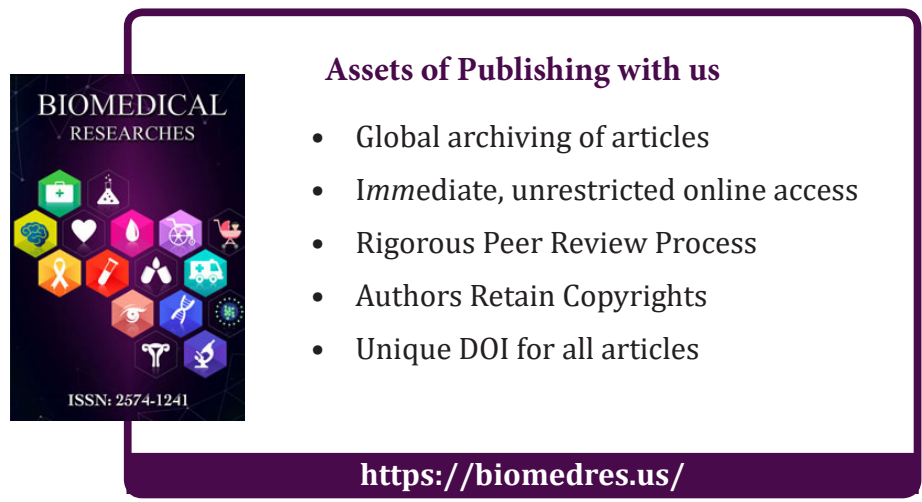

